\title{
Zur Kenntnis von Elektrodenvorgängen. Kontraktometrische Beobachtungen an Anoden
}

von

\author{
V. Kohlsehatter und H. Ståger.
}

(I4. VII. 21.)

Vor einiger Zeit hat der eine von uns (H. Stäger) über Versuche berichtet ${ }^{1}$ ), bei denen das zuerst von Kohlschütter und Vuilleumier ${ }^{2}$ ) benutzte Kontraktometer zur Untersuchung der kathodischen Fällung von Nickelmetall, insbesondere ihrer Beeinflussbarkeit durch Temperatur und Depolarisatoren, verwertet wurde. Die Versuche haben gezeigt, dass durch das einfache Instrument nicht nur empfindlich, sondern vor allem auch völlig eindeutig und zahlenmässig übereinstimmend, Veränderungen angezeigt werden, die sich in dünnsten Elektrodenüberzügen vollziehen. Dies gab den Anlass, das Verfahren auch auf einige Anodenvorgänge anzuwenden, bei denen durch chemische Timwandlung des Elektrodenmetalls entweder festhaftende Schichten einer anderen Substanz offensichtlich entstehen, oder feste Lösungen von Sauerstoff bezw. Sauerstoffverbindungen in der Oberfläche angenommen werden.

Von besonderem Interesse mussten wiederum solche Fälle sein, in denen derartigen Veränderungen, wie dies bei der kathodischen Nickelabscheidung der Fall ist, auffällige Änderungen in den Entladungspotentialen parallel gehen oder zugeschrieben werden. Der Zusammenhang wurde hier teilweise experimentell durch Beobachtung der Klemmenspannung wührend der kontraktometrischen Versuche herzustellen versucht, indern Vorgänge mit unveränderlichen Elektrolyten und kathodischer Wasserstoffentwicklung an einer geschwärzten Platinclektrode benutzt wurden. Man hat dann ein von der Dauer der Elektrolyse nicht beeinflusstes Kathodenpotential; hält man Stromstärke und Temperatur konstant, so ändert sich in dem Ausdrucke für die Badspamung $\mathrm{Bd}=\mathrm{JW}+\mathrm{E}$ das Glied IW nicht, und im Gange der Klemmenspannung ist eine Parallele zum Gange der Anodenspannung gegeben.

1) Helv. 3, 584 (1920).

2) Z. El. Ch. 24, 300 (1918). 
Unter diesen Gesichtspunkten wurden Versuche mit folgenden Anoden und Elektrolyten angestellt: 1. Platin in Natronlauge und Schwefelsäure; 2. Nickel in Natronlauge; 3. Kupfer in Natronlauge; 4. Verschiedene Metalle in Natriumsulfid. -

In erster Linie suchten wir zu ermitteln, ob an sauerstoffentwickelnden ",unangreifbaren“ Anoden mechanisch nachweisbare Veränderungen die bekannten Potentialverschiebungen begleiten, da auf diese Weise der Nachweis vom Auftreten neuer, mehr oder weniger beständiger Stoffarten auf einem unabhängigen Wege und unmittelbar sinnenfällig geführt werden würde.

Die Resultate haben die Erwartung bestätigt. Mechanische Veränderungen der Elektrodenfläche traten auch da deutlich zu Tage, wo sie sonst nicht wahrgenommen werden konnten, während sie in anderen Fällen mit sichtbaren Vorgängen zusammenfielen. Hierdurch wird die Brauchbarkeit der kontraktometrischen Methode als Orientierungsmittel in einem weiten Untersuchungsgebiet erwiesen. Nimmt man noch hinzu, dass solche Veränderungen von dünnen Schichten auch unabhängig von elektrolytischen Vorgängen nachgewiesen werden können, sobald man eine Substanz in dünner Haut auf der Kontraktometerplatte ausbreiten und befestigen kann, so ergibt sich ein allgemeines Verfahren, das für eine spezielle Formart fester Materie der dilatometrischen Methode im Range und in der Anwendbarkeit nicht wesentlich nachsteht. -

Die Verhältnisse sind noch nicht soweit geklärt, dass die Beobachtungsresultate im einzelnen mit den Feststellungen über die Überspannung des Sauerstoffs an Metallelektroden in Beziehung gebracht werden könnten. Wir beschränken uns daher darauf, die Versuchsergebnisse ohne eingehendere Diskussion wiederzugeben; sie untereinander und mit jenen enger zu verknüpfen, wird Sache einer weiteren Verfolgung der Erscheinungen sein.

\section{Sauerstoffpolarisation an Platinanoden.}

Eine Kontraktometerplatte aus blankem Platin wurde bei den Dimensionen, in denen wir sie verwendeten, nicht verändert, wenn sie als sauerstoffentwickelnde Anode bei den für die Versuche benutzten Stromdichten diente. Dagegen erwies sich eine mit einem glatten Platinüberzug versehene Elektrode als ziemlich empfindlich. Die Platinierung wurde durch Elektrolyse einer ca. 0,1-n. Lösung von Platinchlorwasserstoffsäure mit einer Stromdichte von 5 Milliamp. $/ \mathrm{cm}^{2}$ wäh- 
rend 60 Minuten vorgenommen, wonach die Elektrode mit kathodischem Wasserstoff in Schwefelsäure behandelt, sorgfältig ausgewaschen und ohne vorherige Trocknung verwendet wurde. Die Zelle bestand aus einem geschwärzten Platinblech als Kathode und dem Kontraktometer als Anode mit 2-n. Schwefelsäure oder Natronlauge als Elektrolyt. Wurde eine bestimmte Spannung angelegt, so trat sofort nach Stromschluss un rasches Steigen der Klemmenspannung und entsprechendes Sinken der Stromstärke bis zu konstant bleibenden Werten ein, während das Kontraktometer im Sinne einer Dehnung ausschlug. Die letztere ging bei niederen Spannungen zunächst stetig vor sich, bei stufenweiser Steigerung der angelegten Spannung wurde von 0,6 Volt an regelmässig nach ca. einer Minute ein beträchtlicher Sprung in der Zeigerbewegung beobachtet, der eine plötzlich einsetzende Volumen- bezw. Oberflächenvergrösserung anzeigte. Die Erscheinung trat so regelmässig auf, dass an einen Zufall nicht gedacht werden kann. Unmittelbar oder höchstens ganz kurze Zeit nach der sprungweisen Vorwärtsbewegung des Kontraktometerzeigers setzte jedesmal die Sauerstoffentwicklung ein.

Bei Unterbrechung des Stromes fiel die Spannung stossweise ab, während die Anode sich wieder zusammenzog. Mitunter wurde hierbei eine ebenfalls sprungweise Kontraktion beobachtet, doch lag diese nicht an der gleichen Stelle wie bei der Dehnung, sondern trat erst kurz vor Abschluss der Kontraktion auf. Wo der Sprung ausblieb, konnte er • bei Stromumkehr durch Polarisierung mit Wasserstoff sofort erreicht werden; der Zeiger ging dann genau auf die Stelle, die er vor Versuchsbeginn einnahm, zurück. Beobachtungen bei einer bestimmten Anfangsspannung, die nicht nachreguliert wurde, sind in Tab. I und II enthalten. Übersichtlichere Verhältnisse bekommt man natürlich, wenn die Stromstärke konstant gehalten wird. Resultate für je einen Versuch in 2-n. Schwefelsäure und Natronlauge sind in Fig. 1 und 2 zusammengefasst; Tab. III enthält einen Versuch an blankem Platin.

Aus den Figuren ist, in Übereinstimmung mit früheren Untersuchungen anderer Forscher ${ }^{1}$ ), deutlich zu ersehen, dass mit steigender Stromdichte die Anodenspannung schneller und zu höheren Werten ansteigt, und umgekehrt nach Stromunterbrechung langsamer sinkt, wobei bei den höheren Polarisierungen ein ausgesprochen stufenweiser Abfall auftritt. Die miteingetragene Kontraktometerbewegung zeigt den Sprung im Verlauf der Dehnung umso später und umso weniger ausgedehnt, je kleiner die Stromdichte ist.

1) Lit. bei Foerster, Elektrochemie wässriger Lösungen, 2. Aufl., S. 289. - Vgl. insbesondere auch: G. Grube, Z. El. Ch. 24, 237 (1918). 


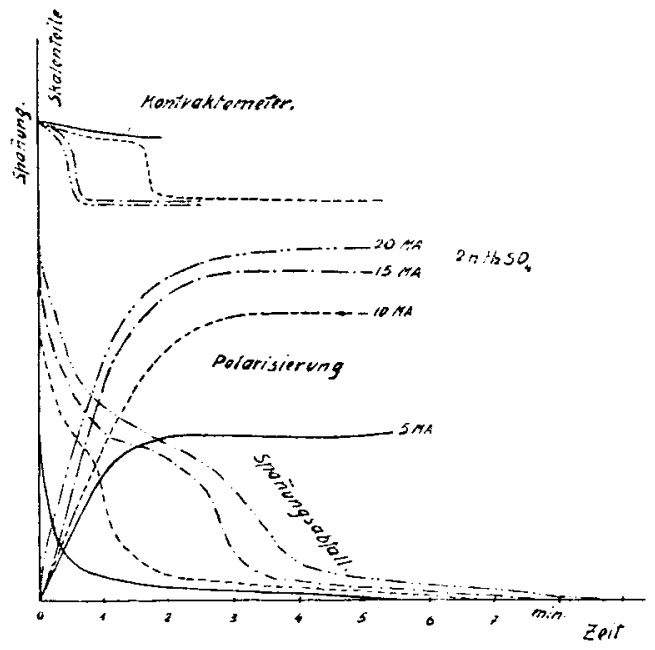

Fig. 1 .

Anstieg der Spannung bei konst. Stromstärke und Kontraktometereffekt. Abfall der Spannung bei Stromlosigkeit.

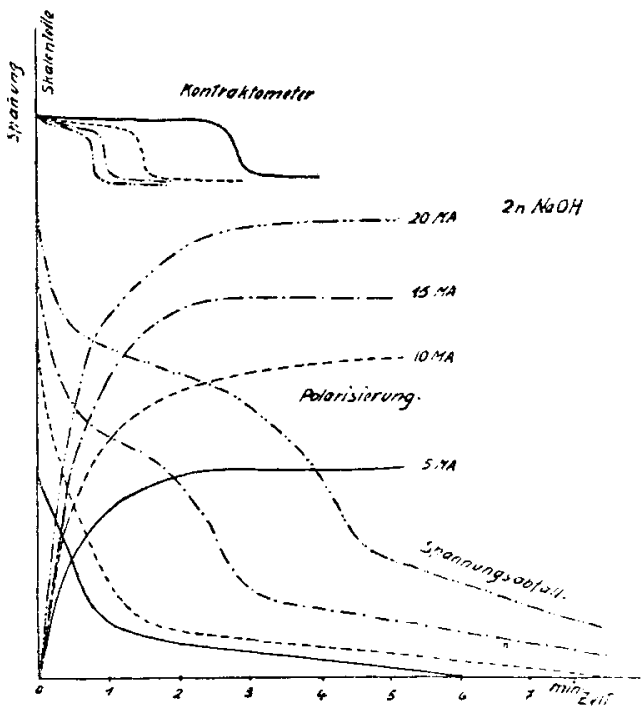

Fig. 2.

Anstieg der Spannung bei konst. Stromstärken.

Kontraktometereffekt. Abfall der Spannung bei Stromlosigkeit. 
Tabelle I.

2 - n. Schwefelsäure.

\begin{tabular}{|c|c|c|cc|}
\hline Zeit & $\begin{array}{c}\text { Spannung } \\
\text { in Volt }\end{array}$ & $\begin{array}{c}\text { Stromstärke } \\
\text { in Milliamp. }\end{array}$ & $\begin{array}{c}\text { Kontraktometer- } \\
\text { stellung }\end{array}$ \\
\hline \hline & 0,20 & 5 & 9,1 & \\
$1^{\prime}$ & 0,24 & 1 & 8,95 & \\
$5^{\prime}$ & 0,24 & 1 & 8,95 & \\
& 0,40 & 12 & 9,10 & \\
$1^{\prime}$ & 0,79 & 5,5 & 8,90 & \\
$2^{\prime}$ & 0,80 & 5,2 & & \\
$5^{\prime}$ & 0,80 & 5,2 & 8,90 & \\
& 0,60 & 26 & 9,10 & \\
$1^{\prime}$ & 1,32 & 15 & 7,70 & Sprung \\
$2^{\prime}$ & 1,40 & 14 & 7,60 & $8,9-7,8$ \\
$3^{\prime}$ & 1,40 & 14 & 7,60 & \\
$5^{\prime}$ & 1,40 & 14 & 7,60 & \\
& 0,80 & 200 & 9,10 & \\
$1^{\prime}$ & 1,74 & 140 & 7,60 & Sprung \\
$2^{\prime}$ & 1,76 & 138 & 7,50 & $8,8-7,7$ \\
$3^{\prime}$ & 1,77 & 137 & 7,40 & \\
$4^{\prime}$ & 1,79 & 132 & 7,30 & \\
$5^{\prime}$ & 1,80 & 130 & 7,30 & \\
\hline
\end{tabular}

Tabelle Ia.

Spannungsabfall nach Stromunterbrechung.

\begin{tabular}{|c|c|c|c|}
\hline Zeit & $\begin{array}{l}\text { Spannung } \\
\text { in Volt }\end{array}$ & \multicolumn{2}{|c|}{ Kontraktometer } \\
\hline & 1,44 & 7,60 & \\
\hline $1 / 4^{\prime}$ & 0,92 & & \\
\hline $1 / 2^{\prime}$ & 0,84 & & \\
\hline $3 / 4^{\prime}$ & 0,80 & 7,70 & \\
\hline $1^{\prime}$ & 0,74 & & \\
\hline $2^{\prime}$ & 0,64 & 7,90 & \\
\hline $3^{\prime}$ & 0,48 & 8,15 & \\
\hline $4^{\prime}$ & 0,12 & 8,20 & \\
\hline $5^{\prime}$ & 0,06 & 8,35 & \\
\hline $10^{\prime}$ & 0,00 & 9,00 & Sprung \\
\hline
\end{tabular}

In 2 -n. Natronlage als Elektrolyt war der Verlauf der Polarisation derselbe. 
Welchem Vorgang, d. h. welchen chemischen oder physikalischen Veränderungen der Effekt zuzuschreiben ist, lässt sich vorläufig nicht mit Sicherheit angeben; vermutet kann aber immerhin werden, dass er auf das zunächst in fester Lösung auftretende, das Potential der Sauerstoffentwicklung vermittelnde unbeständige Oxyd $\mathrm{PtO}_{3}$ zurückgeht, vielleicht auf seine Ausscheidung aus der übersättigten festen Lösung.

Tabelle II.

2 -n. Natronlauge.

\begin{tabular}{|r|c|c|c|}
\hline Zeit & $\begin{array}{c}\text { Spannung } \\
\text { in Volt }\end{array}$ & $\begin{array}{c}\text { Stromstärke } \\
\text { in Milliamp. }\end{array}$ & Kontraktometer \\
\hline & $\mathbf{0 , 2 0}$ & 15 & 9,20 \\
$\mathbf{1}^{\prime} \mathbf{2}^{\prime}$ & 0,70 & 5 & 9,00 \\
$\mathrm{I}^{\prime}$ & 0,70 & 5 & \\
$5^{\prime}$ & 0,70 & 5 & 9,00 \\
& $\mathbf{0 , 4 0}$ & 19 & 9,20 \\
$1^{\prime} \mathbf{2}^{\prime}$ & 0,96 & 8 & 8,95 \\
$\mathbf{1}^{\prime}$ & $\mathbf{1 , 0 2}$ & 7 & 8,90 \\
$2^{\prime}$ & $\mathbf{1 , 0 3}$ & $\mathbf{6 , 5}$ & \\
$3^{\prime}$ & $\mathbf{1 , 0 3}$ & $\mathbf{6 , 5}$ & \\
5 & $\mathbf{1 , 0 3}$ & $\mathbf{6 , 5}$ & \\
& $\mathbf{0 , 6 0}$ & 80 & 9,20 \\
$1^{\prime} \mathbf{2}^{\prime}$ & $\mathbf{1 , 6 0}$ & $\mathbf{5 2}$ & 8,80 \\
$\mathbf{1}^{\prime}$ & $\mathbf{1 , 7 0}$ & $\mathbf{5 1 , 5}$ & $\mathbf{7 , 8 0}$ \\
$2^{\prime}$ & $\mathbf{1 , 7 2}$ & $\mathbf{5 1 , 0}$ & \\
$3^{\prime}$ & $\mathbf{1 , 7 3}$ & $\mathbf{5 0 , 5}$ & $\mathbf{7 , 7 5}$ \\
$\mathbf{5}^{\prime}$ & $\mathbf{1 , 7 3}$ & $\mathbf{5 0 , 5}$ & $\mathbf{7 , 7 5}$ \\
\hline
\end{tabular}

2. Anodische Polarisation an Nichel.

Durch die Untersuchungen von F. Foerster ${ }^{1}$ ) ist festgestellt, dass an Nickelanoden in Alkalilauge, z. B. im Eisen-Nickelsammler, die Sauerstoffentwicklung über die Bildung von höheren Nickeloxyden geht. Es war uns daher von Interesse, zu sehen, ob mit Hülfe des Kontraktometers einzelne Produkte durch mechanische Veränderungen der Anodenfläche bemerkbar gemacht werden können.

$\mathrm{Zu}$ diesem Zwecke wurde eine Versuchsreihe an einem Nickelniederschlag, der aus einer Nickelchloridlösung mit Ammonchlorid als

1) Z. El. Ch. I3, 414 (1907). 


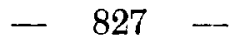

Tabelle III.

Anstieg der Spannung bei konstanter Stromstärke.

2 -n. Natronlauge, Elektrode Pt glatt.

\begin{tabular}{|c|c|c|}
\hline Zeit & $\begin{array}{l}\text { Stromstärke } \\
\text { in Milliamp. }\end{array}$ & Spannung in Volt \\
\hline & 10 & 1,02 \\
\hline $1 / 4^{\prime}$ & & 1,16 \\
\hline $1 / z^{\prime}$ & & 1,20 \\
\hline $1^{\prime}$ & & 1,24 \\
\hline $2^{\prime}$ & & 1,24 \\
\hline $5^{\prime}$ & & 1,26 \\
\hline & 15 & 0,80 \\
\hline $1 / 4^{\prime}$ & & 1,26 \\
\hline $1 / 2$ & & 1,46 \\
\hline $3 / 4^{\prime}$ & & 1,52 \\
\hline $1^{\prime}$ & & 1,64 \\
\hline $\mathbf{2}^{\prime}$ & & 1,58 \\
\hline $5^{\prime}$ & & 1,58 \\
\hline & 20 & 1,00 \\
\hline $1 / 4^{\prime}$ & & 1,48 \\
\hline $1 / 2^{\prime}$ & & 1,62 \\
\hline $3 / 4^{\prime}$ & & 1,63 \\
\hline $1^{\prime}$ & & 1,64 \\
\hline $2^{\prime}$ & & 1,67 \\
\hline $5^{\prime}$ & & 1,68 \\
\hline \multicolumn{3}{|c|}{$\begin{array}{c}\text { Rückgang der Spannung nach } \\
\text { Śtromunterbrechung: }\end{array}$} \\
\hline & 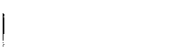 & 1,68 \\
\hline $1 / 4^{\prime}$ & & 0,58 \\
\hline $1 / 2^{\prime}$ & & 0,88 \\
\hline $3 / 4^{\prime}$ & & 0,26 \\
\hline $1^{\prime}$ & & 0,22 \\
\hline $\mathbf{2}^{\prime}$ & & 0,16 \\
\hline $\mathbf{3}^{\prime}$ & & 0,14 \\
\hline $10^{\prime}$ & & 0,00 \\
\hline
\end{tabular}

Zusatz bei einer Stromdichte von 5 Milliamp. $/ \mathrm{cm}^{2}$ während vier Stunden hergestellt war, durchgeführt. Als Elektrolyt wurde im einen Falle eine 6-n. Natronlauge, im andern eine 2-n. Lauge verwendet; als Kathode diente wieder geschwärztes Platin. In den folgenden Tabellen IV und V ist der Gang der Klemmenspannung bei konstanter Stromdichte und der Veränderungen des Kontraktometers wiedergegeben. 
Tabelle IV.

Anstieg der Spannung bei konstanter Stromstärke. 6-n. $\mathrm{NaOH}$

\begin{tabular}{|c|c|c|c|}
\hline Zeit & $\begin{array}{l}\text { Stromstärke } \\
\text { in Milliamp. }\end{array}$ & $\begin{array}{l}\text { Spannung } \\
\text { in Volt }\end{array}$ & Kontraktometer \\
\hline & 5 & 0,64 & 5,6 \\
\hline $1^{\prime}$ & & 0,73 & \\
\hline $10^{\prime}$ & & 0,74 & \\
\hline $30^{\prime}$ & & 0,74 & \\
\hline & 10 & 1,04 & 5,6 \\
\hline $1^{\prime}$ & & 1,18 & \\
\hline $2^{\prime}$ & & 1,26 & \\
\hline $3^{\prime}$ & & 1,29 & \\
\hline $4^{\prime}$ & & 1,30 & \\
\hline $5^{\prime}$ & & 1,31 & \\
\hline $30^{\prime}$ & & 1,31 & \\
\hline & 15 & 1,36 & 5,6 \\
\hline $1^{\prime}$ & & 1,50 & \\
\hline $30^{\prime}$ & & 1,50 & \\
\hline & 20 & 1,40 & 5,6 \\
\hline $1^{\prime}$ & & 1,58 & \\
\hline $30^{\prime}$ & & 1,58 & 5,81 \\
\hline $30^{\prime}$ & & 1,58 & 5,90 \\
\hline & 25 & 1,60 & 5,1 \\
\hline $30^{\prime}$ & & 1,60 & 5,20 \\
\hline $30^{\prime}$ & & 1,60 & 5,45 \\
\hline & 40 & 1,72 & 5,50 \\
\hline $30^{\prime}$ & & 1,72 & 5,65 \\
\hline $30^{\prime}$ & & 1,72 & 5,78 \\
\hline & 100 & 2,12 & 5,78 \\
\hline $30^{\prime}$ & & 2,12 & 5,95 \\
\hline $30^{\prime}$ & & 2,12 & 5,98 \\
\hline
\end{tabular}

Im Verlauf von ca. 1 Minute nach Stromunterbrechung war die Spannung in allen Fällen wieder auf dem Nullpunkte angelangt, doch waren im Abfall immer zwei deutliche Haltepunkte zu beobachten. Die Kontraktion der Nickellamelle ging bei Stromunterbrechung in keinem Falle wieder zur Anfangsstelle zurück, selbst wenn man den Apparat 24 Stunden sich selbst überliess. Die Kontraktion bei der Polarisierung war immer gering und trat nur ein, wo Sauerstoff entwickelt wurde; der Nickelniederschlag zeigte nie eine Verfärbung. 


\section{- 829 \\ Tabelle V.}

Anstieg der Spannung bei konstanter Stromstärke. 2-n. Natronlauge.

\begin{tabular}{|c|c|c|c|}
\hline Zeit & $\begin{array}{l}\text { Stromstärke } \\
\text { in Milliamp. }\end{array}$ & $\begin{array}{l}\text { Spannung } \\
\text { in Volt }\end{array}$ & Kontraktometer \\
\hline & 5 & 0,52 & 5,55 \\
\hline $1^{\prime}$ & & 0,73 & \\
\hline $\mathbf{2}^{\prime}$ & & 0,74 & \\
\hline $\mathbf{3}^{\prime}$ & & 0,75 & \\
\hline $30^{\circ}$ & : & 0,75 & \\
\hline & 10 & 0,56 & 5,90 \\
\hline $1^{\prime}$ & & 1,21 & \\
\hline $2^{\prime}$ & & 1,26 & \\
\hline $3^{\prime}$ & & 1,30 & \\
\hline $4^{\prime}$ & & 1,36 & \\
\hline $5^{\prime}$ & & 1,38 & \\
\hline $30^{\circ}$ & & 1,38 & \\
\hline & 15 & 0,80 & 6,10 \\
\hline $1^{\prime}$ & & 1,44 & \\
\hline $30^{\prime}$ & & 1,44 & \\
\hline & 20 & 1,38 & 6,10 \\
\hline $30^{\prime}$ & & 1,52 & 6,30 \\
\hline $30^{\prime}$ & & 1,52 & $6 ; 35$ \\
\hline & 25 & 1,50 & 6,00 \\
\hline $1^{\prime}$ & & 1,58 & \\
\hline $2^{\prime}$ & & 1,60 & \\
\hline $30^{\prime}$ & & 1,60 & 6,15 \\
\hline $30^{\prime}$ & & 1,60 & 6,20 \\
\hline & 100 & 1,86 & 6,00 \\
\hline $1^{\prime}$ & & 2,04 & \\
\hline $30^{\prime}$ & & 2,04 & 6,15 \\
\hline $30^{\prime}$ & & 2,04 & 6,20 \\
\hline \multicolumn{4}{|c|}{ Rückgang der Spannung, nach Stromunterbruch. } \\
\hline & & 1,30 & \\
\hline $1 / \mathrm{Q}^{\prime}$ & & 0,20 & \\
\hline $1^{\prime}$ & & 0,00 & \\
\hline
\end{tabular}

Angefügt sei noch ein mehr qualitativer kontraktometrischer Versuch mit einem dünnen Nickelniederschlag (aus Nickelchlorid-Ammonchloridlösung mit 5 Milliamp. $/ \mathrm{cm}^{2}$. während einer Stunde hergestellt) in 0,1-n. Natronlauge, welcher zeigt, dass die anodische Oxydation eine gleichmässige hochdisperse Haut erzeugen kann, die in gleicher Weise 
wie eine Metallhaut eine nachträgliche schrumpfung arfährt. Die Kontraktion trat bei einer Stromdichte von 5 Villiamp. $/ \mathrm{cm}^{2}$. ein, während sich die Anode gleichzeitig braun färlte. Beim Lmschalten des Stromes wurde die Elektrode wieder blank und die Kontraktion ging vollständig zurück, wie aus der folgenden Taluelle VI zu entnehmen ist.

Tabelle VI.

Anodische und kathodische Polarisation an eincm Nickelniederschlag.

\begin{tabular}{|c|c|c|c|c|c|}
\hline \multicolumn{3}{|c|}{ Bei Polarisierung } & \multicolumn{3}{|c|}{ Nach Stromunterbrechung } \\
Zeit & $\begin{array}{c}\text { Kontrakto- } \\
\text { meter }\end{array}$ & Differenz & Zeit & $\begin{array}{c}\text { Kiontrakto- } \\
\text { nieter }\end{array}$ & Differenz \\
\hline & 3,70 & & & & \\
$10^{\prime}$ & 4,24 & 0,54 & $10^{\prime}$ & 4,90 & \\
$20^{\prime}$ & 4,42 & 0,18 & $20^{\prime}$ & 4,20 & 0,50 \\
$30^{\prime}$ & 4,60 & 0,18 & $30^{\prime}$ & 4,05 & 0,20 \\
$40^{\prime}$ & 4,73 & 0,13 & $40^{\prime}$ & 3,93 & 0,15 \\
$50^{\prime}$ & 4,82 & 0,09 & $50^{\prime}$ & 3,81 & 0,12 \\
$60^{\prime}$ & 4,90 & 0,08 & $60^{\prime}$ & 3,71 & 0,10 \\
\hline
\end{tabular}

Bei alternierender anodischer und kathodischer Polarisation in Perioden von je zehn Minuten ging der Zeiger immer um gleich viel vorwärts und rückwärts, wobei allerdings mit der Zeit cine gewisse Abnahme in der Bewegungsfähigkeit zu konstatieren war:

\begin{tabular}{|c|c|l|}
\hline Zeit & $\begin{array}{c}\text { Kontrakto- } \\
\text { meter }\end{array}$ & \\
\hline \hline & 2,60 & \\
$10^{\prime}$ & 3,00 & Anole \\
$20^{\prime}$ & 2,60 & Kathode \\
$30^{\prime}$ & 2,90 & Anode \\
$40^{\prime}$ & 2,60 & Kathiode \\
$50^{\prime}$ & 2,80 & Anode \\
\hline
\end{tabular}

Wenn man sich den Vorgang auf der Elektrorle so vorzustellen hat, dass zuerst eine oberflächliche hochdisperse und daher kontraktionsfähige Oxydhaut entsteht, so hat deren Reduktion denselben Effekt, wie die anodische Ablösung eines Metallüberzuges, $d$. h. sie zerstört den Zusammenhang in der kontraktionsfähiren Schicht. Bei erneuter Oxydation wird das auf der Oberfläche verteilt bleibende reduzierte 
Metall zugleich mit solchem aus den in Zwischenräumen seiner Teilchen liegenden tieferen Schichten oxydiert; durch Wiederholung der Oxydation und Reduktion wird allmählich eine Vergröberung des Überzugs eintreten und die Kontraktionsfähigkeit nach und nach abnehmen, wie es der Versuch zeigt.

\section{Verhalten von Kupferanoden in Natronlauge.}

In clen Zusammenhang der in dieser Mitteilung beschriebenen Beobachtungen gehören einige Versuche, die bereits unter anderen Gesichtspunkten von Kohlschütter und Tüischer ${ }^{1}$ ) behandelt wurden und hier nur kurz erwähnt werden sollen.

An einer Kupferanode in Natronlauge lassen sich nach E. Müller ${ }^{2}$ ) durch Verfolgung des Potentials verschiedene Zustände nachweisen, die dadurch von besonderem Interesse sind, dass die Vorgänge, durch die sie veranlasst werden, sich deutlich unterscheiden und mit Sicherheit angeben lassen. Geht man von blankem Kupfer aus, so entstehen in scharf abgesetzten Stufen nacheinander Cupro-oxyd, Cuprihydroxyd, bezw. Cuprioxyd und Kupferperoxyd, was sich in einem diskontinuierlichen Spannungsanstieg sehr charakteristisch ausdrückt; der Zeitpunkt, zu dem dieser einsetzt, hängt von der Konzentration der Lauge und der Stromdichte ab.

Lm die Wirkung der auf der Anode zweifellos auftretenden Deckschichten zu verfolgen, wurde eine Kontraktometerplatte von denselben Dimensionen wie die sonst verwendeten Platinlamellen benutzt, die zwar nicht die gleiche Elastizität wie diese besass, aber, wie schon Kohlschiitter und Vuilleumier nachgewiesen haben, durchaus verwendl,ar war. Wegen der Einzelheiten der Versuche mit dieser Platte sei auf die angeführte Arbeit verwiesen. Während einer nach anfänglichem raschem Spannungsanstieg gleichmässig sich vollziehenden Elektrolyse, die zur Lösung von Kupfer als Cuprihydroxyd führte, wurde eine stetige Kontraktion angezeigt; unter anderen Bedingungen, bei denen die Stromdichte und Konzentration der Lösung einen plötzlichen Sprung in der Spannung verursachte und der Anodenvorgang in Sauerstoffentwicklung endigte, fand anfänglich auch eine schwache aber stetige Kontraktion der Elektrode statt, die jedoch genau bei dem Spannungssprung ein Maximum erreichte und dann langsam zurückging. Offenbar reranlasst also die Hydroxydbildung eine Kontraktion der Anoden-

1) Z. an. Ch. III, 193 (1920).

2) Z. El. Ch. 13, 133 (1907). 
oberfläche, während die Bildung von Peroxyd, las als Vermittler der Sauerstoffentwicklung auftritt, bezw. die letztere selbst eine Dehnung bedingt.

Diesen Versuchen wurden einige andere mit zwei elektrolytischen Kupferniederschlägen auf dem Platinkontraktometer angefügt.

Von den Niederschlägen war der eine (I) aus schwefelsaurer Sulfatlösung, der andere aus alkalischer weinsäurehaltiger Lösung (II) erzeugt worden. Ihre Verschiedenheit trat nicht nur im Aussehen hervor, indem ersterer fahlrot und unter dem Mikroskop' schuppig erschien, letzterer dunkler und strukturlos war, sondern auch im Gang der Kontraktion bei der kathorlischen Bildung, wie sich ans folgenden Daten ergibt.

Tabelle VII.

Bildung der Kupferniederseh!äge.

\begin{tabular}{|r|c|c|c|c|c|}
\hline \multicolumn{2}{|c|}{ I. } & Schwefelsaure Lösung. & \multicolumn{3}{|c|}{ II. Alkalische Lösung. } \\
\hline Zeit & $\begin{array}{c}\text { Kontrakto- } \\
\text { meter }\end{array}$ & Diff. 2' & Zeit & $\begin{array}{c}\text { Kontrakto- } \\
\text { meter }\end{array}$ & Diff. 2' \\
\hline \hline & 0,50 & & & 0,10 & \\
$2^{\prime}$ & 1,17 & 0,67 & $2^{\prime}$ & 1,00 & 0,90 \\
$4^{\prime}$ & 1,42 & 0,25 & $4^{\prime}$ & 2,00 & 1,00 \\
$6^{\prime}$ & 1,63 & 0,21 & $6^{\prime}$ & 3,10 & 1,10 \\
$8^{\prime}$ & 1,77 & 0,14 & $8^{\prime}$ & 4,00 & 0,90 \\
$10^{\prime}$ & 1,86 & 0,09 & $10^{\prime}$ & 5,00 & 1,00 \\
$12^{\prime}$ & 1,94 & 0,08 & $12^{\prime}$ & 6,20 & 1,20 \\
$14^{\prime}$ & 1,99 & 0,05 & $14^{\prime}$ & 7,40 & 1,20 \\
$16^{\prime}$ & 2,04 & 0,05 & $16^{\prime}$ & 8,50 & 1,10 \\
$18^{\prime}$ & 2,08 & 0,04 & $18^{\prime}$ & 9,70 & 1,20 \\
$20^{\prime}$ & 2,12 & 0,04 & $20^{\prime}$ & 10,70 & 1,00 \\
\hline
\end{tabular}

Als diese Niederschläge in 0,1-n. Natronlauge bei einer Stromdichte von 5 Milliamp. $/ \mathrm{cm}^{2}$ polarisiert wurden, trat bei I sofort Dunkelfärbung der Oberfläche und Bildung dunkler Oxydschlieren auf, wiahrend die Platte sich langsam streckte; bei II kam es erst nach einiger Zeit zur Abscheidung von Oxyd in der Flüssigkeit und die Durchbiegung ging im ganzen nur wenig zurück. Die beobachtete Dehnung war offenbar nur durch eine tcilweise Ablösung der Kupferschicht, durch deren Bildung das Kontraktometer gespannt war, verursarht, und ebenso dïrfte die geringe Streckung der Elektrode zu beurteilen scin, die bei einigen Versuchen mit Kupferniederschlägen in reinem Wasser unter Anlegung einer Spannung von 72 Volt in dit Erscheinung trat. 
Die letzteren Versuche wurden mit Rücksicht auf eine vor einiger Zeit von Kohlschiitter ${ }^{1}$ ) angegebene neue Methode der elektrischen Kolloïdsynthese angestellt, die darauf beruht, dass auf einer Anode durch elektrolytischen Stromfluss in reinem Wasser eine Oxydschicht erzeugt und dauernd durch eine dem elektrolysierenden Strom übergelagerte schnellschwingende Entladung zerstäubt wird. Es kamen die beiden Arten von Kupferniederschlägen zur Verwendung, jeder in einer bezw. drei Stunden mit 5 Milliamp. $/ \mathrm{cm}^{2}$ hergestellt. Die Erscheinungen waren im Wesentlichen gleich: zu Beginn der Elektrolyse trat keine Gasentwicklung auf, vielmehr schied sich vor der Anode etwas schwammiges bezw. flockiges Hydroxyd ab, das sich alsbald in fein verästelten Formen in der Richtung der Kraftlinien einstellte und zur Kathode hinüberwuchs; danach setzte Gasentwicklung ein, indem sich zugleich die Anode dunkel färbte. Die Vorgänge verlaufen daher qualitativ offenbar denen in Natronlauge analog.

\section{Verhalten von Eisenanoden in Natronlauge.}

Im Hinblick auf die Passivierung, die Eisen bei anodischer Polarisierung erfahren kann, haben wir einige Versuche über das Verhalten einer mit Eisen bedeckten Kontraktometerplatte in Natronlauge angestellt. Die Eisenniederschläge wurden aus einer 20-proz. Eisensulfatlösung, der 5\% Magnesiumsulfat und etwas Natriumbicarbonat zugesetzt war, mit 2 Milliamp. $/ \mathrm{cm}^{2}$ in 1 bezw. 3 Stunden erzeugt; sie zeigten unter dem Mikroskop eine gleichmässige dichte Struktur.

In 0,1-n. Natronlauge mit 2 Milliamp. $/ \mathrm{cm}^{2}$ polarisiert gaben beide Anoden sofort Gasentwicklung, während sich eine geringe Kontraktion bemerkbar machte; sie betrug z. B. bei einem einstündigen Niederschlag 0,3 , bei einem dreistündigen 0,58 bezw. 0,71 Teilstriche in 30 Minuten, bei nachfolgender kathodischer Polarisation ging die Kontraktion ein wenig $\left(0,1\right.$ Teilstrich in $\left.30^{\prime}\right)$ zurück. Die Niederschläge lösten sich nach der anodischen Behandlung nur schwer in Salzsäure, waren also deutlich passiv und blieben es auch, wenn sie nachträglich zur Kathode gemacht worden waren.

Zwei Versuche mit dreistündigen Niederschlägen nahmen folgenden Verlauf:

a) Das Kontraktometer wurde zuerst während 10 Minuten zur Kathode gemacht, wobei eine Dehnung um 0,3 Teilstriche eintrat; beim Ausschalten des Stromes ging der Zeiger um 0,6 Teilstriche in 10

1) Z. El. Ch. 25, 309 (1919). 
Minuten über seine Anfangsstellung hinaus. Erneute Wasserstoffbeladung mit Stromdichte $=10$ Milliamp. $/ \mathrm{cm}^{2}$ hatte wierler eine Dehnung um 0,7 Teilstriche zur Folge, die bei Stronunterbrechung zurückging.

b) Abwechselnde anodische und kathodische Polarisierung gab folgende Kontraktionen und Dehnungen (Tabelle VIII):

Tabelle VIII.

\begin{tabular}{|r|c|c|}
\hline Zeit & Kontraktometer & Kontraktometer \\
\hline 1. & Anodische Polarisation & Kathodische Polarisation \\
$2^{\prime}$ & 3,69 & 4,04 \\
$10^{\prime}$ & 3,90 & 3,81 \\
& 4,04 & 3,73 \\
$2^{\prime}$ & 3,73 & 4,08 \\
$10^{\prime}$ & 3,93 & 3,84 \\
& 4,08 & 3,75 \\
$2^{\prime}$ & 3,75 & \\
$10^{\prime}$ & 3,94 & \\
& 4,09 & \\
Anstieg der Klemmenspannung & von 1.65 auf 1,85 Volt. \\
2. & Kathodische Polarisation & Anotische Polarisation \\
& & 4,90 \\
$2^{\prime}$ & 5,30 & 5,10 \\
$10^{\prime}$ & 5,03 & 5,13 \\
$30^{\prime}$ & 4,98 & 5,15 \\
$60^{\prime}$ & 4,90 & 5,15 \\
$120^{\prime}$ & 4,90 & 5,15 \\
$150^{\prime}$ & 4,90 & 5,30 \\
$180^{\prime}$ & - & - \\
\hline & 4,90 & \\
\hline
\end{tabular}

5. Verhalten von chemisch passiviertem Eisen.

Nachdem sich wenigstens Andeutungen über einen kontraktometrischen Effekt an anodisch-polarisiertem Eisen ergeben hatten, wurde versucht, Strukturänderungen auch an dünnen Eisenschichten, die der Einwirkung eines starken Oxydation $s$ mittels ausgesetzt wurden, nachzuweisen.

Bekanntlich wird, wie zuerst Schönbein gefunden hat, Eisen durch Eintauchen in konzentrierte Salpetersäure in der Art passiv, dass es sich nicht in der Säure löst und auch gegenülıer anderen Kationen nicht seine normale elektromotorische Wirksamkeit betïtigt; erst mit der Zeit, 
oder dureh plöt:zliche mechanische Erschätterung wird es in den Stand gesetzt, edleres Metall niederzuschlagen.

Die benutzten Eisenniederschläge wurden in sechs Stunden mit 5 Milliamp. $/ \mathrm{em}^{2}$ auf der Kontraktometerlamelle er\%eugt und ohne Kombination mit einer zweiten Elektrode verwendet.

Wurde das Kontraktometer mit der Eisenschicht in Salpetersäure $(1,4)$ eingetaucht, so trat regelmässig ein nur einige sekunden anhaltendes leichtes Aufbrausen ein, während die Platte eine kleine Dehnung durch $A$ blösung von Metall erfuhr $(0,5,0,95,0,4$ 'Teilstriche hei 3 Versuchen). Der Eisenüberzug wurde silberblank, veränderte sich aber nicht weiter, wenn er in der Salpetersäure verblieb; der Zeiger blieb kurze Zeit stchen und zeigte dann eine langsame Kontraktion an, \%. B.:

$\begin{array}{ccccc} & 10^{\prime} & 30^{\prime} & 60^{\prime} & 90 \\ 4,9 & \mathbf{5}, 1 & 5,35 & \mathbf{5 , 5 7} & 5,70\end{array}$

Nach dem Einbringen in IVasner gring die Kontraktion nicht weiter; wurde las Wasser gegen Kupfersulfatlösung ersetzt, so blieb der Zeiger zunächst ebenfalls stehen, nach einigen Minuten begann jedoch eine Dehnung der Platte bis zur vollständigen Streckung, indem das Eiven sich löste und ein nur lose haftender Niederschlag von Kupfer an seine Stelle trat.

Bei cinem anderen Versuch wurde die Platte sofort nach der Passivierung des Fisens durch die Salpetersäure in Wasser gebracht. Auch liee fand eine langsame Kontraktion statt:

$\begin{array}{llll} & 30^{\prime} & 60 & 90^{\prime} \\ \mathbf{4 , 9 5} & 5,23 & 5,30 & 5,30\end{array}$

Beim Eintauchen in Kupfersulfat sehied sich wïhrend etwa жwi Minuten kein Netall aus, dann wurde Kupfer unter langsamer Entspannung der Platte in gleichmässig dichter Schicht niedergesehlagen; der Eintritt der Abscheidung kann durch mechanische Erschütterung beschleunigt werden, die form des Kupferniederschlags richtet sich nach der form des Eisens.

In einem lalle zeigte sich ein eigenartiges Verhalten, das für die Beurteilung der an orler in der Eisenschicht sich abspielenden Vorgänge wichtig erscheint: Eis machte sich ein Schwanken bezw. IIn- und Hergehen des Zeigers bemerkbar, das sich nur auf kleine $A$ bstände erstreckte, aber bei der Sicherheit, mit der das Kontraktometer sonst dem Sinne nach reagiert, anzeigt, dass sich offenbar zwei Vorgänge mit entgegengesetzter IFirkung abspielen. Nach der ersten Dehnung beim 
Eintauchen in Salpetersäure $(0,4$ Teilstriche) spielte der Zeiger längere Zeit mit einer Amptitude von 0,2 Teilstrichen, bis die Bewegung langsam. in die einer Kontraktion entsprechende Richtung überging (0,35 Teilstriche in 25 Minuten); in Wasser eingetauch1 trat nochmals eine schwache Dehnung und erneute Kontraktion ein; in verdünnter Salzsäure setzte sich diese noch fort, der Niederschlag nahm eine lunkle Farbe an und löste sich unter Dehnung der Platte langsam ab.

An Nickelschichten, die mit oder ohne Wechselstromüberlagerung hergestellt waren ${ }^{\mathbf{1}}$ ) zeigte sich bei nachfolgender Behandlung mit konzentrierter Salpetersäure keine kontraktometrisch nachweisbare $\ddot{\Lambda}$ derung; die mit Wechselstrom erzeugten lösten sich nicht, die anderen langsam unter gleichzeitiger Entspannung der Platte.

\section{Verhalten von Metallanoden in Sulfidlösungen.}

Zum Schlasse seien noch einige Versuche wiedergegeben, bei denen dünne Sulfidüberzüge auf ihr Verhalten geprült werden sollten; sie wurden angestellt, weil gerade Sulfide leicht in Gestalt metallähnlicher Häute erhalten werden können und anzunehmen war, dass an solchen strukturelle Veränderungen mit der Zeit verfolgbar sein würden.

Die Niederschläge wurden durch Elektrolyse in verdünnter Sulfidlösung erzeugt, die aus 0,1-n. Natronlauge durch Sättiguug mit Schwefelwasserstoff und Zufügen der gleichen Menge Natronlauge hergestellt war. Die Metalle, die anodisch mit ihr behandelt wurden, waren auf der Platinlamelle niedergeschlagen.

Von den untersuchten Metallen lieferten Nickel und Kupfer, das aus saurer Lösung gefällt war, keine festhaftenden Sulfidüberzüge, Kupfer aus alkalischer Lösung gab bei Anwendung einstündiger Niederschläge in der Sulfidlösung mit 5 Milliamp. $/ \mathrm{cm}^{2}$ nur eine Dehnung des: Platte durch Zerstörung der zusammenhängenden Mctallschicht, obwohl der feinkörnige und gleichmässige Uberzug selbst festhaftete. Zweistündige Niederschläge gaben zuerst eine schwache Kontraktion, weiterhin eine Dehnung, z. B.:

\begin{tabular}{l|l|ll} 
& 4,60 & & 1,60 \\
$10^{\circ}$ & 4,90 & $11^{\prime}$ & 1,80 \\
$20^{\prime}$ & 4,10 & $19^{\prime}$ & 0,80
\end{tabular}

Die besten Resultate wurden mit Silberschichten aus cyankalischer Lösung erhalten. (0,5-n. $\mathrm{AgNO}_{3}, 2$-n. $\mathrm{KCX} ; 5$ Milliamp./cm² ${ }^{2}$ ) Auch

1) Vgl, die Mitteilung von Stäger 1. c. 
hier zeigte sich ein deutlicher Unterschied zwischen dünnen und dickeren Schichten (einstündige und dreistündige Niederschläge), indem die ersteren in 0,1-n. und 0,01-n. Natriumsulfid eine gleichmässige Kontraktion erfuhren, während sich die dreimal so dicken Schichten zuerst dehnten, um sich im weiteren Verlauf der Sulfidbildung ebenfalls zu kontrahieren. In Fig. 3 sind die Resultate zur Darstellung gebracht.

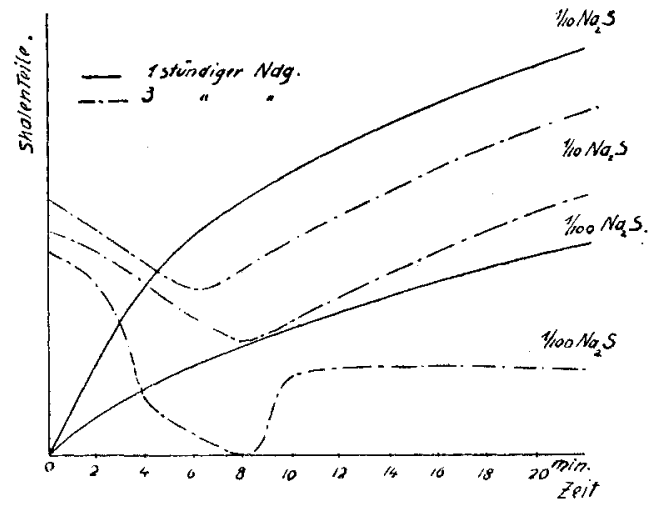

Fig. 3.

Anodische Silbersulfidbildung.

Die Erklärung dürfte sein, dass sich bei den dickeren Schichten zuerst eine oberflächliche zusammenhängende aber doch hochdisperse Haut von Silbersulfid bildet, das mehr Platz beansprucht, als das zu seiner Bildung verwendete Metall, so dass eine Dehnung eintritt. Wenn die Sulfidbildung tieferliegende Schichten ergreift, kann auch eine vertikal zur Platte gerichtete Teilchenvergrösserung erfolgen, die eine durchgreifende Schrumpfung oder Sinterung der Sulfidschicht und damit die Kontraktion bedingt; bei dünnen Silberschichten tritt die letztere unmittelbar ein.

Hinsichtlich der allgemeinen Bedeutung der Erscheinungen, die bei den kontraktometrischen Beobachtungen zu Tage treten, sei auf das Nachwort von $V$. Kohlschütter zu der ersten Mitteilung von $H$. Stäger verwiesen.

Bern, Anorgan. Laboratorium der Universität. 